\title{
The frequency response of the object, and the estimation of the parameters of the simplified transfer function model
}

\author{
Konrad Dudziak ${ }^{1 *}$, Krzysztof Stawicki ${ }^{2}$, and Andrzej Brykalski ${ }^{2}$ \\ ${ }^{1}$ West Pomeranian University of Technology, Faculty of Civil Engineering and Architecture, al. Piastów 50, 70-311 Szczecin, Poland \\ ${ }^{2}$ West Pomeranian University of Technology, Faculty of Electrical Engineering, Sikorskiego 37, 70-313 Szczecin, Poland
}

\begin{abstract}
The article presents a comparison of the modified method of the moments and the System Identification Toolbox ${ }^{\mathrm{TM}}$ for the purpose of determining equivalent parameters (depending on the space point, time constants, delays, row of inertia) of simplified transfer function models.
\end{abstract}

\section{Introduction}

Simplified transfer function models are widely used in automation, electromagnetic fields, temperature fields. These models can be used to describe the dynamics of diffusion processes. The simplified model is a model described by lower order transfer function than the order of the base model. In practical applications, simplified models are used of second, third or fourth order.

Determining the parameters of transfer function models from the frequency response is not an easy task. In practice, there are few tools and methods that allow this [1], namely: transfer function spectral approximation method (Rao-Lamba method); method of approximation of real and imaginary parts of the nominator and denominator of spectral transfer function (Reddy's method); method of the moment and modified (by the authors) method of the moment (MM) [2,3].

One of the few existing tools that allows to determine the parameters of transfer function models is the System Identification Toolbox ${ }^{\mathrm{TM}}(\mathrm{SIT})$ from the MATLAB ${ }^{\circledR}$ package.

This paper presents a comparison of the quality of simplified models obtained using the modified method of the moment and the System Identification Toolbox TM.

\section{Calculations}

In this work, the following transfer function models were compared:

$$
\frac{k_{0}}{1+s \tau}(\mathrm{MT} 1), \frac{k_{0} \exp \left(-s \tau_{0}\right)}{1+s \tau} \text { (MT2), } \frac{k_{0}}{\left(1+s \tau_{1}\right)\left(1+s \tau_{2}\right)} \text { (MT3) }
$$

The results obtained with MM and SIT were compared with the reference results obtained in simulations in the COMSOL Multiphysics $\AA$ program, which uses the finite element method (FEM) for the appropriate Helmholtz equation. The simulation was carried out in frequency domain. The measurement point located inside the screen $(r=0 \mathrm{~mm}, z=40 \mathrm{~mm})$ was selected for the comparative analysis.

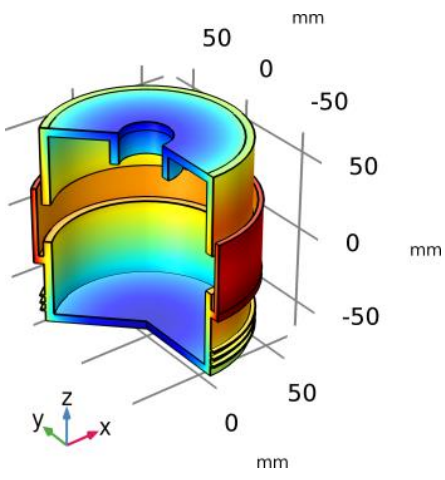

Fig. 1. The tested can screen model.

The model was placed in a homogeneous field directed along the $z$ axis.

In order to determine the parameters of transfer function models (1) using the MM, it is necessary to determine the first two normalized moments $M_{1}$ and $M_{2}$ from the formulas (2)(3).

$$
\begin{gathered}
M_{1}=-\frac{1}{P(0)} \frac{\sum_{i=1}^{N} \omega_{i}^{6} \sum_{i=1}^{N} \omega_{i} Q\left(\omega_{i}\right)-\sum_{i=1}^{N} \omega_{i}^{4} \sum_{i=1}^{N} \omega_{i}^{3} Q\left(\omega_{i}\right)}{\sum_{i=1}^{N} \omega_{i}^{2} \sum_{i=1}^{N} \omega_{i}^{6}-\left(\sum_{i=1}^{N} \omega_{i}^{4}\right)^{2}} \\
M_{2}=-\frac{2}{P(0)} \frac{\sum_{i=1}^{N} \omega_{i}^{8} \sum_{i=1}^{N} \omega_{i}^{2}\left[P\left(\omega_{i}\right)-P(0)\right]-\sum_{i=1}^{N} \omega_{i}^{6} \sum_{i=1}^{N} \omega_{i}^{4}\left[P\left(\omega_{i}\right)-P(0)\right]}{\sum_{i=1}^{N} \omega_{i}^{4} \sum_{i=1}^{N} \omega_{i}^{8}-\left(\sum_{i=1}^{N} \omega_{i}^{6}\right)^{2}}
\end{gathered}
$$

The error of approximation were defined as:

$$
\delta=\int_{0}^{f_{g}}\left[B_{z}(f)-B(f)\right]^{2} d f
$$

where: $B_{z}(f)$ - values of the $B_{z}$ component of the magnetic flux density vector obtained from models with estimated parameters (MM or SIT),

$B(f)$ - reference values of the $B_{z}$ component of the magnetic flux density vector obtained from the COMSOL Multiphysics ${ }^{\circledR}$ program (FEM),

$f_{g}$ - limit frequency.

Figures 2, 4, 6 present frequency characteristics of MT1, MT2, MT3 models, respectively. 


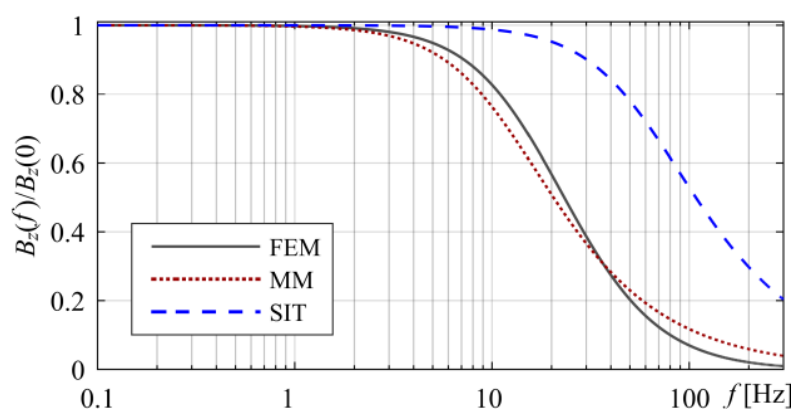

Fig. 2. Frequency characteristics of the MT1 model.

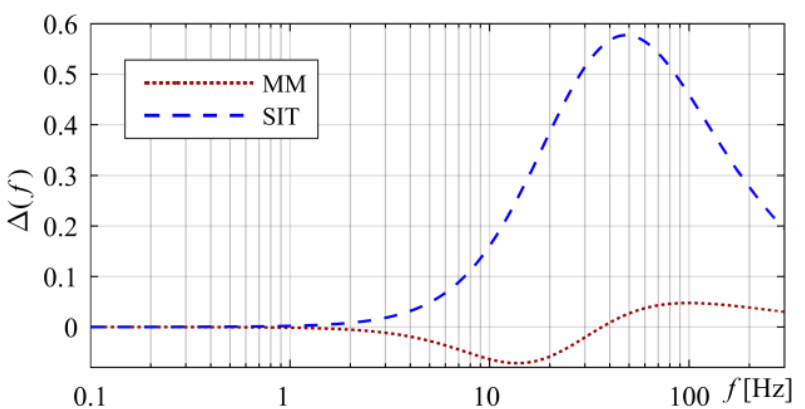

Fig. 3. MT1 model matching error.

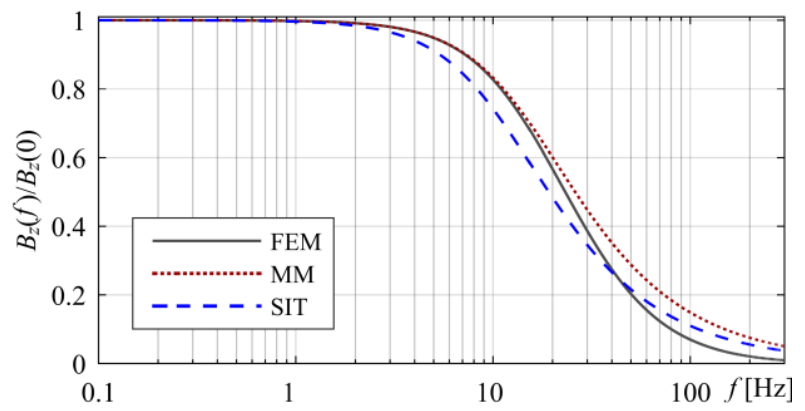

Fig. 4. Frequency characteristics of the MT2 model.

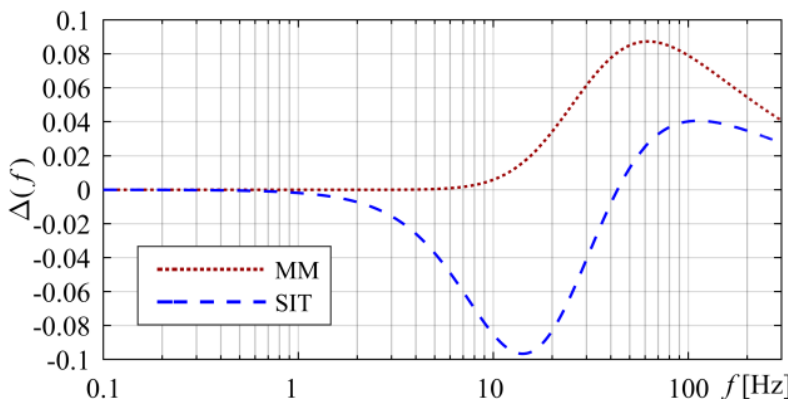

Fig. 5. MT2 model matching error.

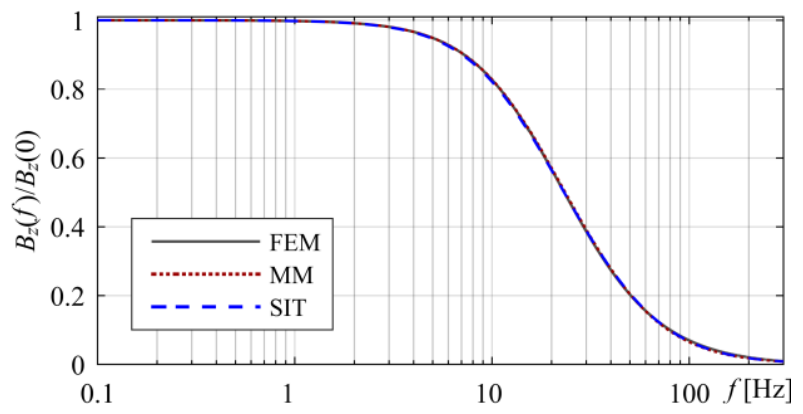

Fig. 6. Frequency characteristics of the MT3 model.

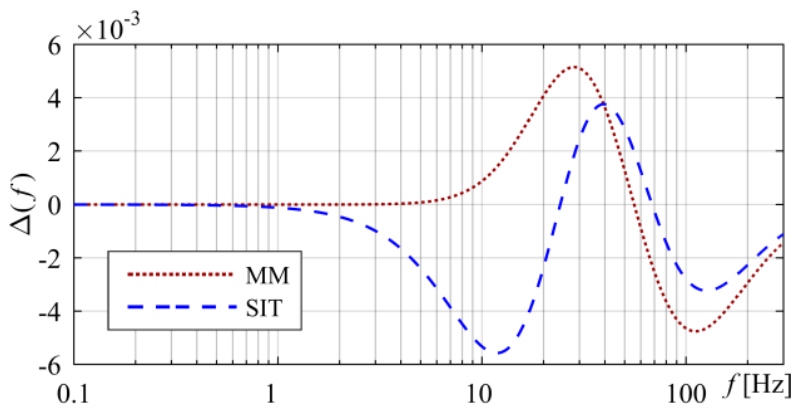

Fig. 7. MT3 model matching error.

Table 1 shows the numerical results of the error calculated in accordance with equation (4). Lower value, better model fit.

Table 1. Error $\delta$ calculated from the equation (4).

\begin{tabular}{|c|c|c|}
\hline $\begin{array}{c}\text { Transfer } \\
\text { function model }\end{array}$ & $\begin{array}{c}\text { Modified method } \\
\text { of the moment } \\
\text { (MM) }\end{array}$ & $\begin{array}{c}\text { System Identification } \\
\text { Toolbox }^{\text {TM }} \text { (SIT) }\end{array}$ \\
\hline MT1 & 2,68 & 69,52 \\
\hline MT2 & 2,18 & 6,49 \\
\hline MT3 & 0,06 & 0,07 \\
\hline
\end{tabular}

\section{Conclusions}

The results of the research show that both the MM and SIT enable quite correct approximation of the parameters of simplified transfer function models. However SIT was not able to determine the equivalent time constant for the MT1 (see Figures 2 and 3) which strongly differ from the reference results (FEM). For the MT2 and MT3 models both methods give similar and acceptable results, but MM produce smaller or much smaller errors (4). It is worth to note that the MM has one major advantage over SIT. It allows easy implementation in other algorithms (including in source codes). Numerical experiments have shown that estimation of simplified transfer function parameters on the basis of complex frequency characteristics proved to be more effective not only in the frequency domain (expected result) but also in the time domain (i.e. [3]).

\section{References}

[1] J. Halawa, Transactions of the Society for Computer Simulation, (USA), Vol. 6, No. 1, pp. 17-29, 1989.

[2] K. Dudziak, K. Stawicki, A. Brykalski, International Interdisciplinary PhD Workshop (IIPhDW), 2018, pp. 151-155.

[3] K. Dudziak, K. Stawicki, A. Brykalski, Poznan University of Technology Academic Journals: Electrical Engineering, no. 97, pp. 17-28, 2019. 